\title{
SCIENTIFIC REPORTS

\section{Mutant IDH1 confers resistance to energy stress in normal biliary cells through PFKP-induced aerobic glycolysis and AMPK activation}

\author{
Hiroaki Fujiwara ${ }^{1,2}$, Keisuke Tateishi ${ }^{1 *}$, Kento Misumi ${ }^{3}$, Akimasa Hayashi ${ }^{3}$, Kaori Igarashi ${ }^{4}$, \\ Hiroyuki Kato ${ }^{1}$, Takuma Nakatsuka ${ }^{1}$, Nobumi Suzuki ${ }^{1}$, Keisuke Yamamoto ${ }^{1}$, Yotaro Kudo ${ }^{1}$, \\ Yoku Hayakawa ${ }^{1}$, Hayato Nakagawa ${ }^{1}$, Yasuo Tanaka ${ }^{1}$, Hideaki ljichi ${ }^{1}$, Hirofumi Kogure ${ }^{1}$, \\ Yosuke Nakai ${ }^{1}$, Hiroyuki Isayama ${ }^{6}$, Kiyoshi Hasegawa ${ }^{5}$, Masashi Fukayama ${ }^{3}$, \\ Tomoyoshi Soga ${ }^{4} \&$ Kazuhiko Koike ${ }^{1}$
}

Metabolism is a critical regulator of cell fate determination. Recently, the significance of metabolic reprogramming in environmental adaptation during tumorigenesis has attracted much attention in cancer research. Recurrent mutations in the isocitrate dehydrogenase (IDH) 1 or 2 genes have been identified in several cancers, including intrahepatic cholangiocarcinoma (ICC). Mutant IDHs convert $\alpha$-ketoglutarate ( $\alpha-K G)$ to 2 -hydroxyglutarate (2-HG), which affects the activity of multiple $\alpha$-KGdependent dioxygenases including histone lysine demethylases. Although mutant $I D H$ can be detected even in the early stages of neoplasia, how IDH mutations function as oncogenic drivers remains unclear. In this study, we aimed to address the biological effects of IDH1 mutation using intrahepatic biliary organoids (IBOs). We demonstrated that mutant IDH1 increased the formation of IBOs as well as accelerated glucose metabolism. Gene expression analysis and ChIP results revealed the upregulation of platelet isoform of phosphofructokinase-1 (PFKP), which is a rate-limiting glycolytic enzyme, through the alteration of histone modification. Knockdown of the Pfkp gene alleviated the mutant IDH1-induced increase in IBO formation. Notably, the high expression of PFKP was observed more frequently in patients with IDH-mutant ICC compared to in those with wild-type IDH ( $p<0.01,80.9 \%$ vs. $42.5 \%$, respectively). Furthermore, IBOs expressing mutant IDH1 survived the suppression of ATP production caused by growth factor depletion and matrix detachment by retaining high ATP levels through $5^{\prime}$ adenosine monophosphate-activated protein kinase (AMPK) activation. Our findings provide a systematic understanding as to how mutant IDH induces tumorigenic preconditioning by metabolic rewiring in intrahepatic cholangiocytes.

Intrahepatic cholangiocarcinoma (ICC) is the second most common liver cancer with an overall poor prognosis ${ }^{1}$. Due to the limited effectiveness of drugs, the five-year survival rate remains less than $10 \%{ }^{2}$. Primary risk factors of ICC include: infection by liver flukes or hepatitis virus, primary sclerosing cholangitis, and intrahepatic stones ${ }^{3}$. Although whole genomic sequencing has identified the mutational landscape of $\mathrm{ICC}^{4,5}$, its pathogenesis remains unclear.

The mutations in isocitrate dehydrogenase 1 (IDH1) or isocitrate dehydrogenase 2 (IDH2) genes are identified in $10-30 \%$ of ICC. Specifically, the mutations are found in ICC without the infection of liver flukes or hepatitis

\footnotetext{
${ }^{1}$ Department of Gastroenterology, Graduate School of Medicine, The University of Tokyo, 7-3-1 Hongo, Bunkyo-ku, Tokyo, 113-8655, Japan. ²Division of Gastroenterology, The Institute for Adult Diseases, Asahi Life Foundation, 2-2-6 Bakurocho, Chuo-ku, Tokyo, 103-0002, Japan. ${ }^{3}$ Department of Pathology, Graduate School of Medicine, The University of Tokyo, 7-3-1 Hongo, Bunkyo-ku, Tokyo, 113-8655, Japan. ${ }^{4}$ Institute for Advanced Biosciences, Keio University, 246-2 Kakuganji, Tsuruoka, Yamagata, 997-0052, Japan. ${ }^{5}$ Hepato-Biliary-Pancreatic Division, Department of Surgery, Graduate School of Medicine, The University of Tokyo, 7-3-1 Hongo, Bunkyo-ku, Tokyo, 113-8655, Japan. ${ }^{6}$ Department of Gastroenterology, Graduate School of Medicine, Juntendo University, 2-1-1 Hongo, Bunkyo-ku, Tokyo, 113-8421, Japan. *email: ktate-tky@umin.ac.jp
} 
virus $^{6,7}$. They are often mutually exclusive with mutations in Kirsten rat sarcoma viral oncogene homolog (KRAS) and tumor protein p53 (TP53) genes ${ }^{8,9}$. ICC with IDH mutation have specific features such as DNA hypermethylation or distinct drug sensitivity ${ }^{10-12}$. Typically, IDH is a metabolic enzyme that catalyzes the oxidative decarboxylation of isocitrate to $\alpha$-ketoglutarate $(\alpha-\mathrm{KG})$ and $\mathrm{CO}_{2}$ during the citric acid cycle ${ }^{11}$. In mutant IDH, a specific metabolite $\mathrm{R}(-)$-2-hydroxyglutarate (2-HG) is produced from $\alpha-\mathrm{KG}^{12}$. 2-HG inhibits the family of $\alpha-K G-d e p e n d e n t$ dioxygenase enzymes, including lysine histone demethylases (KDM) and the ten-eleven translocation (TET) family of DNA hydroxylases ${ }^{13,14}$. The aberrant epigenetic regulation caused by IDH mutation is associated with abnormal cellular differentiation and/or carcinogenesis ${ }^{15,16}$.

Mutant IDH was reported to affect the initiation of Grade II/III gliomas or acute myeloid leukemia ${ }^{15-17}$. Although ICC originates from several cell lineages including hepatocytes, cholangiocytes or, liver progenitor cells $^{18,19}$, how IDH mutation contributes to oncogenic processes of ICC is still unknown. A recent study showed that mutant IDH inhibits hepatocyte nuclear factor 4-alpha (HNF-4 $\alpha)$ expression to preferentially induce biliary lineage cells from liver progenitors, resulting in ICC formation combined with Kras mutations in mice ${ }^{20}$. Recently, organoid techniques have made it possible to culture normal epithelial cells derived from primary tissues ${ }^{21-23}$. In this study, we established mouse intrahepatic biliary organoids (IBOs) that expressed mutant IDH1 to elucidate the functional role of mutant IDH1 in biliary tumorigenesis.

\section{Results}

IDH1 mutation enhances the formation of biliary organoids established from murine liver. To elucidate how mutant IDH affects the molecular or biological features of normal intrahepatic cholangiocytes, we established IBOs from murine normal liver cells (Fig. 1A and Supplementary Fig. 1A) ${ }^{24}$. Given that organoids maintained the characteristics of primary cells ${ }^{24}$, we applied this system to estimate the biological and metabolic traits triggered by IDH1 mutation in normal biliary epithelial cells. IBOs were composed of a monolayer of biliary lineage cells expressing biliary marker genes, including cytokeratin 7 (Ck7), cytokeratin 19 (Ck19), and hepatocyte nuclear factor 1-beta $(H n f 1 \beta)$, but not hepatocyte marker genes such as transthyretin (Ttr) and aldolase B $(A l d o B)$ (Fig. 1A and Supplementary Fig. 1A). Immunohistochemistry confirmed the expression of CK19 and SRY (sex determining region Y)-box 9 (SOX9) as biliary marker proteins in IBOs (Fig. 1B). The established IBOs were transduced with lentiviral vectors expressing human wild-type or mutant IDH1 (R132C) (Supplementary Fig. 1B). The activity of transduced mutated IDH1 was confirmed by production of 2-HG (Fig. 1C). We found that IBOs expressing mutant IDH1 (mut-IBOs) exhibited enhanced organoid-forming efficiency compared to IBOs expressing wild-type IDH1 (wt-IBOs), constitutively through serial passages (Fig. 1D, E). The 2-HG addition also enhanced the formation of IBOs after the third passage (Fig. 1F). In addition, a specific inhibitor of mutant IDH1 ${ }^{25}$, AGI-5198, significantly reduced the 2-HG production and hampered the formation of mut-IBOs. The effects were specific for mut-IBOs because AGI-5198 did not affect the growth of wt-IBOs (Fig. 1G,I). These results indicated that mutant IDH1 has the biological potential to enhance the IBO forming ability.

Mutant IDH1 upregulates glucose metabolism in IBOs. To uncover metabolic traits underlying the increased forming ability in mut-IBOs, metabolic profiles of the IBOs were analyzed by capillary electrophoresis-mass spectrometry (CE-MS) (Fig. 2A and Supplementary Fig. 2). Notably, mut-IBOs displayed enrichment of glycolytic intermediates including fructose-1,6-bisphosphate (F1,6 P), 3-phosphoglycerate (3PG), 2-phosphoglycerate (2PG), phosphoenolpyruvate (PEP), and lactate compared with wt-IBO (Fig. 2A). In addition, the metabolites of other glucose-utilizing pathways sedoheptulose-7-phosphate (S7P), adenosine monophosphate (AMP), uridine monophosphate (UMP) in the pentose phosphate pathway (PPP), and UDP-N-acetylglucosamine in the hexosamine biosynthesis pathway (HBP), were significantly accumulated in mut-IBOs (Fig. 2A). Consistent with an increase in glycolysis, glucose uptake was significantly enhanced in mut-IBOs compared to wt-IBOs (Fig. 2B). Given that all of these metabolic profiles were identified under aerobic culture conditions with sufficient glucose and glutamine, such preferred aerobic glycolysis seemed to be similar to the 'Warburg effect' - a well-known hallmark of rapidly proliferating mammalian cells ${ }^{26}$. However, some metabolites in the TCA cycle, including citrate, fumarate, and malate, were also elevated in mut-IBOs (Supplementary Fig. 2), indicating that mutations in IDH1 lead to higher glucose metabolism. Since reprogramming from differentiated cells to cancer cells is often accompanied by aerobic glycolytic phenotypes, we aimed to investigate if activated glycolysis correlates with the enhanced formation of mut-IBOs by treatment with a glycolytic inhibitor, 2-deoxy-d-glucose (2DG) (Fig. 3A). Although 2DG reduced the organoid-forming efficiency of both wt- and mut-IBOs in a dose-dependent manner, the formation of mut-IBOs was relatively resistant to the inhibitory effects of 2DG compared to wt-IBOs (Fig. 3B,C). The forming capacity of mut-IBOs compared to wt-IBOs was relatively conserved under a low glucose condition (Fig. 3D,E). These results suggest that the formation of IBOs depends on glucose as a critical energy source, and IDH1 mutation allows for IBOs to become resistant to glucose restriction.

Mutant IDH1 activates glycolysis through the upregulation of Pfkp gene. To address the molecular mechanisms underlying increased glycolysis in mut-IBO, we performed microarray gene expression analysis involving $>40,000$ oligonucleotide probes. Regardless of the drastic changes in organoid-forming efficiency and metabolism by IDH1 mutation, the analysis showed that only 19 genes were upregulated $>2$-fold, and 27 genes were downregulated $<2$-fold in mut-IBOs compared to wt-IBOs $(\mathrm{P}<0.05$, Supplementary Fig. 3A and Supplementary Table 1). Consistent with the effects of IDH1 mutation in terms of catalytic activities of $\alpha \mathrm{KG}$-dependent histone lysine demethylases, the levels of global histone $\mathrm{H} 3$ lysine 4 trimethylation (H3K4me3) and lysine 27 trimethylation (H3K27me3) were elevated in mut-IBOs, compared to wt-IBOs (Supplementary Fig. 3B) as previously reported in cancer cells ${ }^{15,16}$. Expression of genes encoding enzymes such as Jarid la, Jarid $1 \mathrm{~b}$, or Jmjd3 were not affected (Supplementary Fig. 3C). In contrast, there were no significant differences in the levels 
A

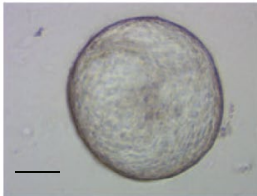

B

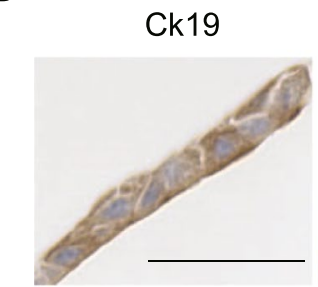

D

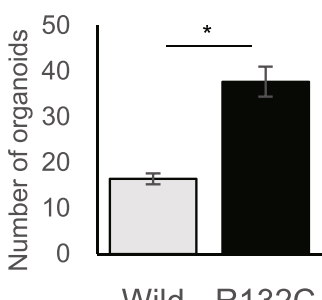

Wild R132C

E

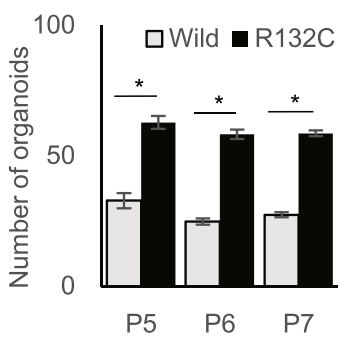

Sox9

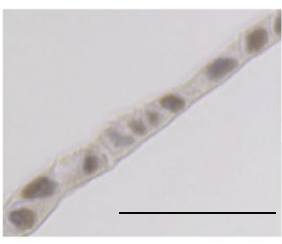

Wild

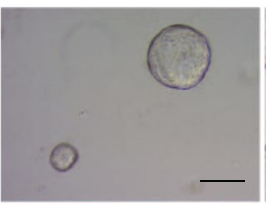

F
C

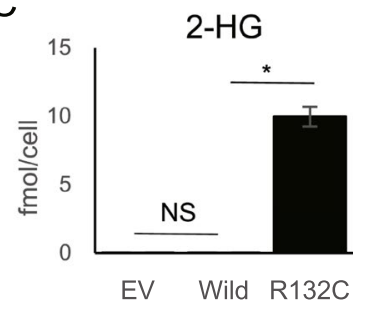

G

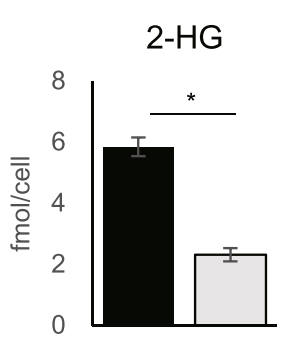

(-) $\quad(+)$

$\mathrm{H}$

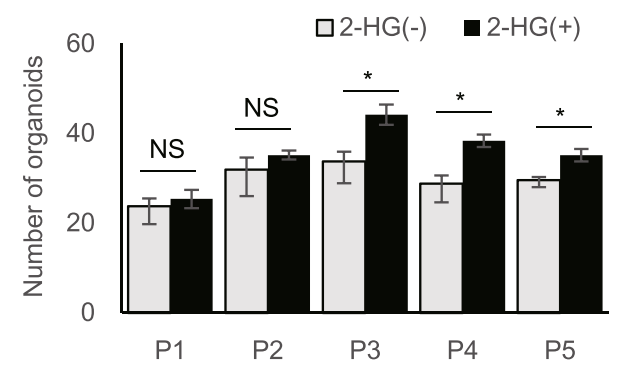

R132C

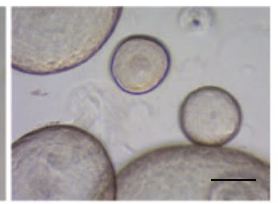

।

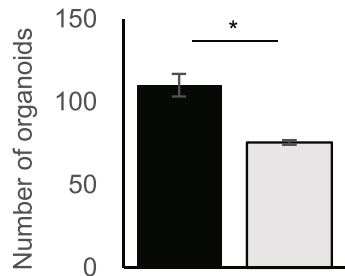

$(-) \quad(+)$

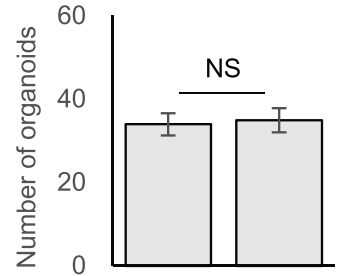

$(-) \quad(+)$

AGI-5198

AGI-5198

Figure 1. IDH1 mutation enhances the formation of biliary organoids established from murine liver. (A) The left panel shows a representative image of IBOs from wild-type mice (8-weeks old, male) at day 7. The middle and left panels show hematoxylin and eosin (HE) staining of them. Scale bars, $250 \mu \mathrm{m}$ (middle and left panels) and $25 \mu \mathrm{m}$ (right panel). (B) Immunohistochemical staining of Ck19 and Sox9 in IBOs. Scale bars, $20 \mu \mathrm{m}$. (C) The amount of 2-HG measured by CE-MS in cell extracts from IBOs stably expressing empty vector (EV), wild-type (Wild), and mutant IDH1 (R132C). ( $\mathrm{n}=4, * \mathrm{P}<0.05$, NS not significant). (D) The number of IBOs $(>100 \mu \mathrm{m})$ at $7 \mathrm{~d}$ after plating $(\mathrm{n}=4,3,000$ cells per group, $* \mathrm{P}<0.05)$ and their representative images. Scale bars, $250 \mu \mathrm{m}$. The assay was performed at passage 4. (E) Organoid-forming efficiency of the indicated IBOs during serial passage (P5-7) after puromycin selection. The number of organoids $(>100 \mu \mathrm{m})$ at $7 \mathrm{~d}$ after plating $(\mathrm{n}=4,3,000$ cells per group, $* \mathrm{P}<0.05)$. (F) IBOs established from wild-type mice treated with $10 \mathrm{mM} 2-\mathrm{HG}$ $(+)$ or vehicle $(-)$ upon serial passage. The number of organoids $(>100 \mu \mathrm{m})$ at $7 \mathrm{~d}$ after plating $(\mathrm{n}=5,3,000$ cells per group, $* \mathrm{P}<0.05$, NS not significant). (G) $2-\mathrm{HG}$ levels of mut-IBOs treated with $20 \mu \mathrm{M}$ AGI-5198 $(+)$ or DMSO vehicle $(-)$. $(\mathbf{H}, \mathbf{I})$ The number $(>100 \mu \mathrm{m})$ of mut-IBOs $(\mathbf{H})$ and wt-IBOs $(\mathbf{I})$ treated with $20 \mu \mathrm{M}$ AGI$5198(+)$ or DMSO vehicle at $7 \mathrm{~d}$ after plating $(\mathrm{n}=4,10,000$ cells per group, $* \mathrm{P}<0.05)$. 
A

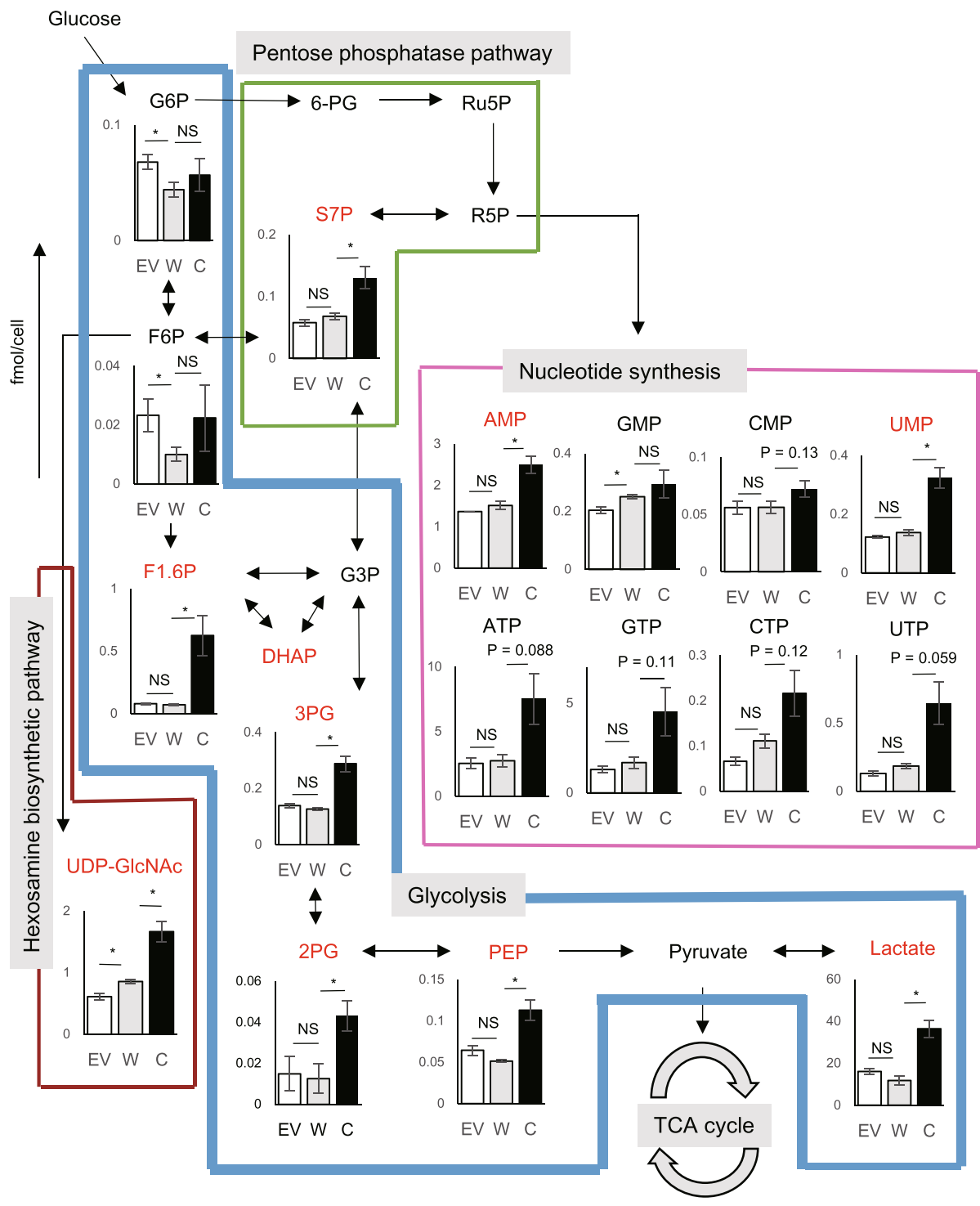

B

Glucose Uptake

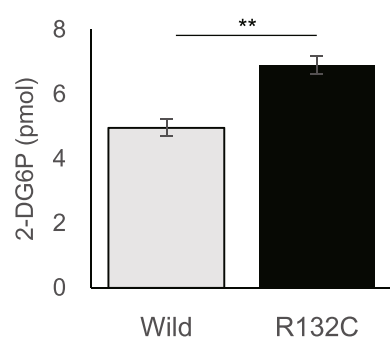

Figure 2. Mutant IDH1 increases glycolytic flux in IBOs. (A) Metabolome analysis of IBOs stably expressing empty vector (EV), wild-type IDH1 (Wild) or mutant IDH1 (R132C) by CE-MS ( $\mathrm{n}=4, * \mathrm{P}<0.05$, NS not significant). (B) Glucose uptake in the indicated IBOs $(\mathrm{n}=3, * \mathrm{P}<0.05)$. Abbreviation; $\alpha-\mathrm{KG}, \alpha-k e t o g l u t a r a t e ;$ AMP, adenosine monophosphate; ATP, adenosine triphosphate; CMP, cytidine monophosphate; CTP, cytidine triphosphate; DHAP, dihydroxyacetone phosphate; F1,6P, fructose-1,6-bisphosphate; F6P, fructose 6-phosphate; GMP, guanosine monophosphate; G3P, glyceraldehyde 3-phosphate; G6P, glucose-6-phosphate; GTP, guanosine triphosphate; 6-PG, 6-phosphogluconate; PEP, phosphoenolpyruvate; 3PG, 3-phosphoglycerate; 2PG, 2-phosphoglycerate; R5P, ribose 5-phosphate; Ru5P, ribulose 5-phosphate; S7P, sedoheptulose-7phosphate; UDP, uridine diphosphate UDP-GlcNAc, uridine diphosphate $\mathrm{N}$-acetylglucosamine; UMP, uridine monophosphate; UTP, uridine triphosphate. 
A

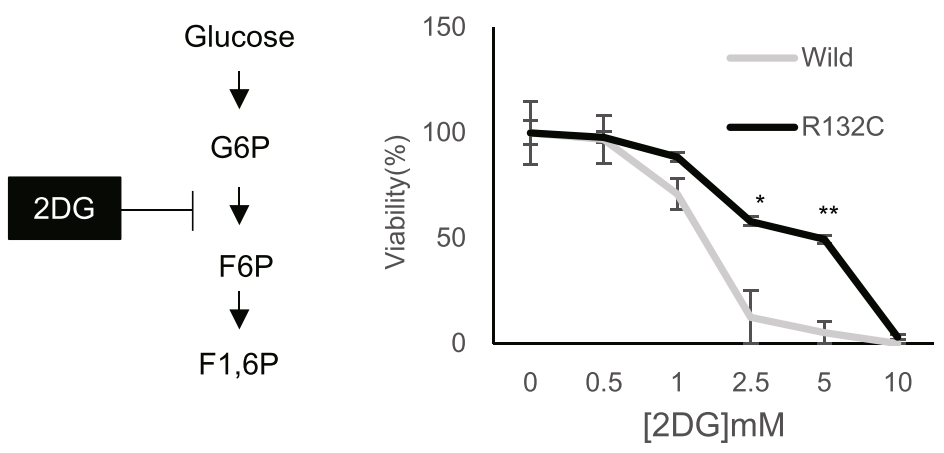

C

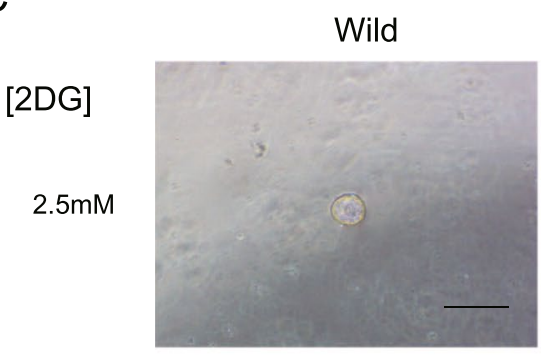

$\mathrm{D}$

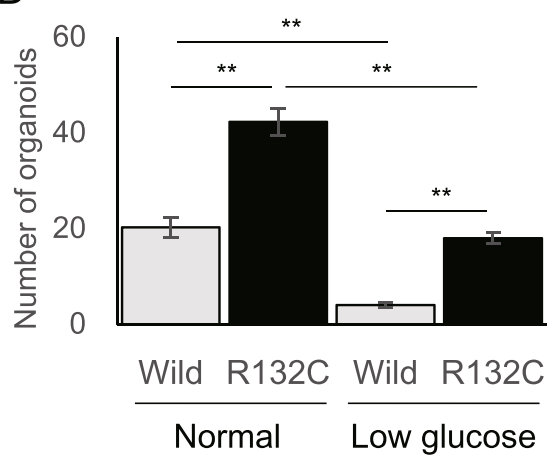

$\mathrm{R} 132 \mathrm{C}$

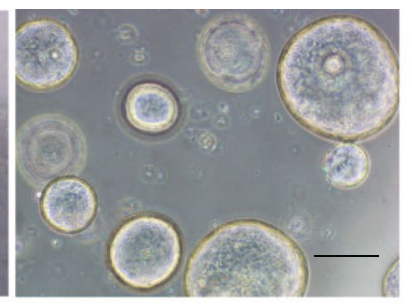

$E$

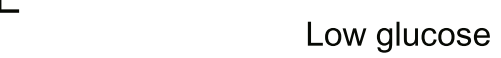

Wild
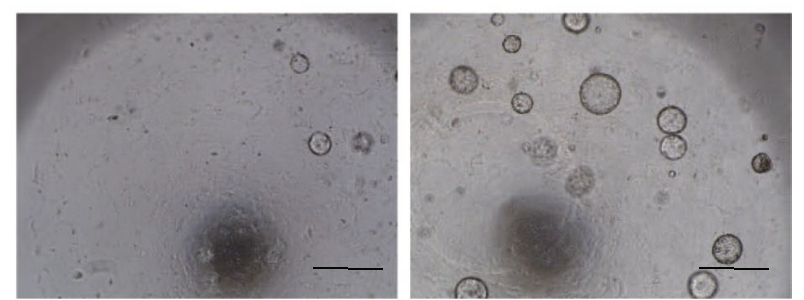

Figure 3. The glycolytic inhibitor 2-DG or glucose restriction hampers the formation of IBOs, and mut-IBOs were resistant to the inhibition. (A) 2-DG competitively inhibits the conversion of G6P to F6P in glycolysis. (B) The number of organoids $(>100 \mu \mathrm{m})$ at $5 \mathrm{~d}$ after plating in medium with or without 2DG (normalized to that in the 2DG-free medium, $\mathrm{n}=4,5,000$ cells per group $* \mathrm{P}<0.05$ ). (C) Representative images. Scale bars, $250 \mu \mathrm{m}$. (D) The number of IBOs $(>100 \mu \mathrm{m})$ was counted at $7 \mathrm{~d}$ after plating under the normal culture condition (Normal) and the low glucose condition (Low glucose), replacing advanced-DMEM/F12 (3 g/L glucose) to L0091, glucose-free DMEM/F12 instead ( $\mathrm{n}=4,5,000$ cells per group, $* * \mathrm{P}<0.01)$. Low glucose medium contains a little glucose derived from Wnt3a- and RSPO1-conditioned medium; the final concentration of glucose is $0.2 \mathrm{~g} / \mathrm{L}$. (E) Representative images. Scale bars, $500 \mu \mathrm{m}$.

of 5-hydroxymethylcytosine (5-hmC), 5-methylcytosine (5-mC), or in the expression of Tet family genes encoding DNA demethylase (Supplementary Fig. 3C-E). In addition, we analyzed the effects on chromatin structure caused by mutant IDH1 using an assay for transposase-accessible chromatin using sequencing (ATAC-seq) ${ }^{27}$. Although different peak patterns were detected in certain locations, there were no significant differences in specific motif sequences binding transcription factors (TFs) between wt- and mut-IBOs (Supplementary Fig. 4A-C). These results appear to be compatible with the lack of differences in DNA methylation, and the limited number of differentially expressed genes between wt- and mut-IBOs. Notably, however, we found a significant upregulation in the expression of the platelet isoform of phosphofructokinase-1 (Pfkp) gene in mut-IBOs (Fig. 4A). Mammalian phosphofructokinase-1 (PFK-1) is a tetramer consisting of three isoforms; Pfkl, Pfkm, and Pfkp ${ }^{28}$ and catalyzes the conversion of fructose 6-phosphate (F6P) to F1,6 P, which is a rate-limiting step in the glycolytic pathway. Intriguingly, the promotion of glycolysis in mut-IBOs occurred immediately downstream the transition of F6P to F1,6 P (Fig. 2A). This led us to hypothesize that IDH1 mutation drives glycolysis through the upregulation of Pfk-1 activity. Supporting our hypothesis, we confirmed not only increases in Pfkp mRNA and protein levels but also identified elevated Pfk-1 activity in mut-IBOs (Fig. 4A-D). To analyze if PFKP contributes to IBO formation, we established two distinct mut-IBO lines in which Pfkp was stably knocked-down (Fig. 4E). Both mut-IBO lines decreased the efficiency of IBO formation by mutant IDH1 (Fig. 4F), emphasizing the notion 
A

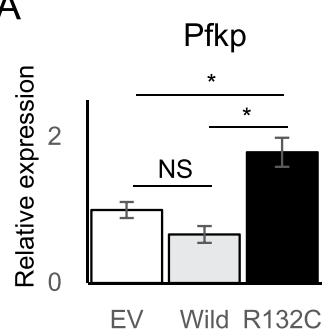

$\mathrm{E}$

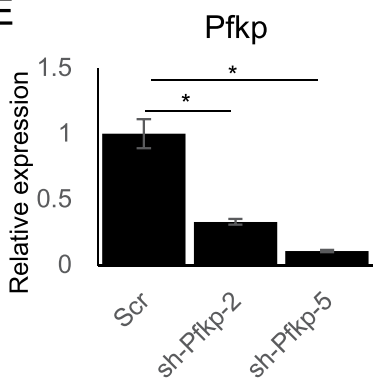

B

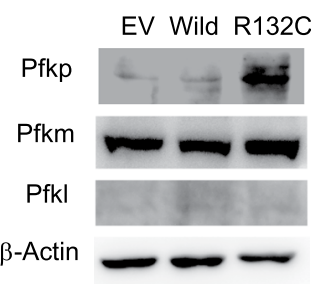

C

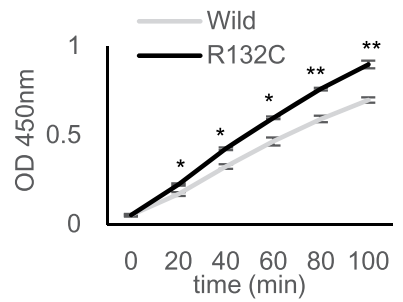

D

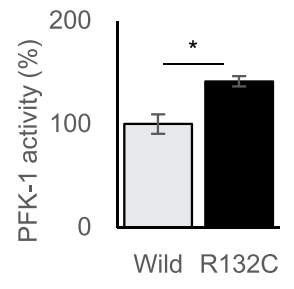

$\mathrm{F}$

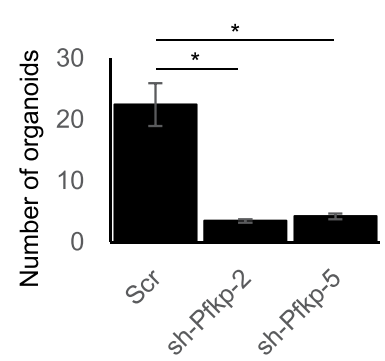

G

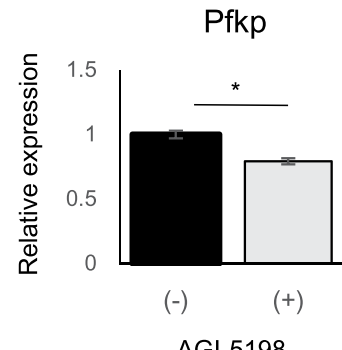

AGI-5198

$\mathrm{H}$

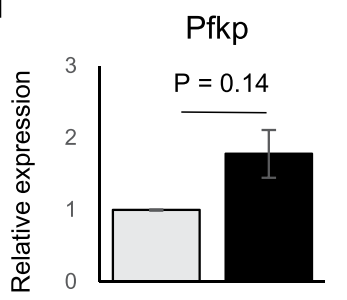

$(-)$

I
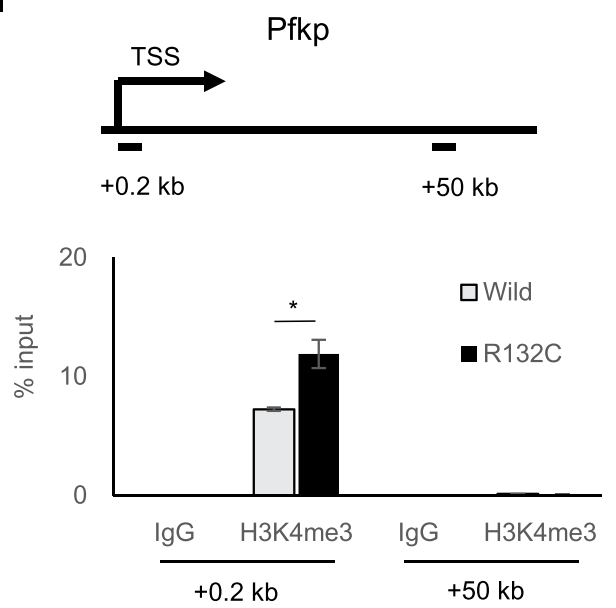

Figure 4. Mutant IDH1 activates glycolysis in IBOs through the upregulation of $P f k p$. (A) The level of $P f k p$ expression in the indicated IBOs by RT-qPCR $(\mathrm{n}=4$, $* \mathrm{P}<0.05$, NS not significant). (B) Protein expression levels of Pfkp, Pfkm, and Pfkl as determined by western blotting. (C,D) Enzymatic assay of PFK-1 activity in the indicated IBOs. Time course of optical absorbance at $450 \mathrm{~nm}$ in (C), and comparison of PFK-1 activity in (D) $(\mathrm{n}=4, * \mathrm{P}<0.05, * * \mathrm{P}<0.01)$. (E) Gene expression of Pfkp in mut-IBOs transfected with shRNA for Pfkp (shPfkp-2, 5) or scrambled shRNA (Scr) as control $(\mathrm{n}=4, * \mathrm{P}<0.05)$. (F) Organoid-forming efficiency of the indicated IBOs $(>100 \mu \mathrm{m})$ at 7 days after plating $(\mathrm{n}=4,3000$ cells per group, $* \mathrm{P}<0.05)$. (G) Gene expression of Pfkp in mut-IBOs treated with $20 \mu \mathrm{M}$ AGI-5198 (+) or DMSO vehicle $(-)(\mathrm{n}=4$, *P $<0.05)$. (H) IBOs established from wild-type mice were treated with $10 \mathrm{mM} 2-\mathrm{HG}(+)$ or vehicle $(-)$ upon serial passage. Gene expression of $P f k p$ at passage 4 by RT-qPCR $(n=3, P=0.14)$. (I) The level of trimethylation of histone $\mathrm{H} 3$ lysine4 (H3K4me3) on the promoter region of Pfkp by ChIP analysis in IBOs. The upper panel shows the schematic diagram of the transcriptional start site (TSS) and amplicons $(0.2 \mathrm{~kb}$ and $50 \mathrm{~kb}$ downstream of the TSS). The lower graph shows ChIP- qPCR data (relative to input DNA, $\mathrm{n}=4$, $* \mathrm{P}<0.05$ ).

that IDH1 mutation promotes IBO formation by enhancing glycolysis via upregulation of PFKP. To examine the dysregulation of $P f k p$ directly caused by $I D H 1$ mutation, the effect of the inhibitor AGI-5198 on the expression of the Pfkp gene was analyzed ${ }^{25}$. AGI-5198 decreased the expression of $P f k p$ in mut-IBOs (Fig. 4G), and, in turn, 2-HG treatment increased the expression of $P f k p$ in IBOs from normal mouse liver (Fig. $4 \mathrm{H}$ ), suggesting that the upregulation of $P f k p$ was attributed to $I D H 1$ mutation. In addition, although ATAC-seq data showed comparable accessibility surrounding the $P f k p$ locus, the level of $\mathrm{H} 3 \mathrm{~K} 4 \mathrm{me} 3$ as an active transcriptional mark was significantly increased on the promoter region of Pfkp in mut-IBOs compared to wt-IBOs (Supplementary Fig. 4D and Fig. 4I), indicating that the upregulation of $P f k p$ was mediated by aberrant histone modification. These data indicated that the epigenetic disturbance induced by $I D H 1$ mutation was focally limited to specific targets, such as $P f k p$ in normal biliary cells. Furthermore, we investigated the expression status of PFKP protein in 101 surgically resected ICC specimens from our hospital. PFKP expression was detected in 69 cases (68\%) (Fig. 5A,B), and the expression levels were significantly higher in ICCs harboring IDH mutations $(\mathrm{P}<0.01$, Fischer's exact test $)$ 
A

Negative (score 0/1) Low (score 2)
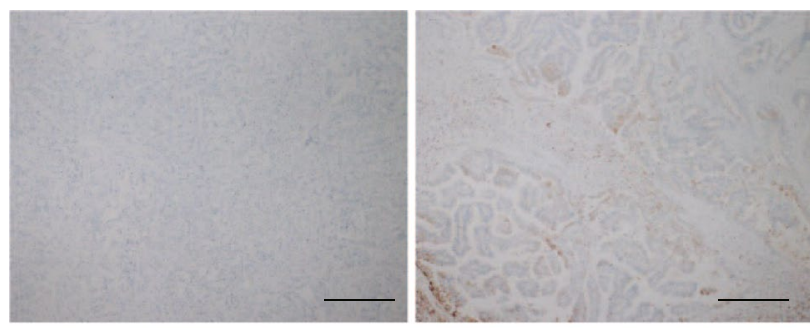

Moderate (score 3)

High (score 4)
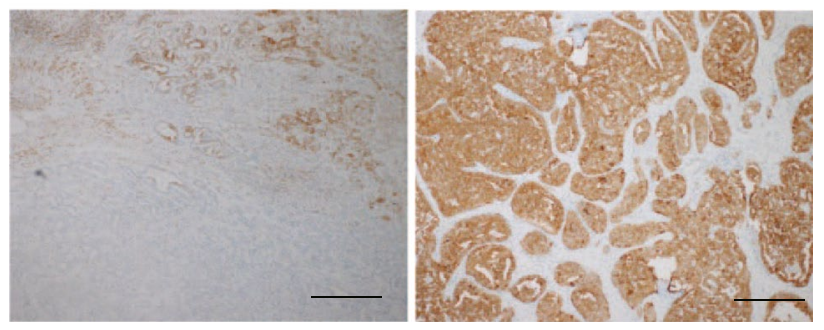

B

\section{PFKP expression}

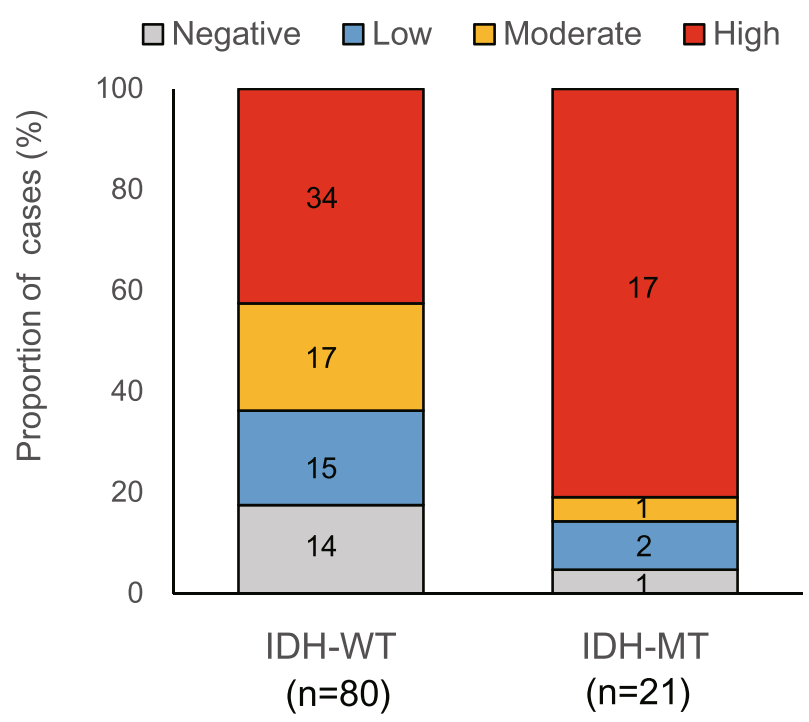

Figure 5. PFKP shows a high expression in IDH-mutant ICCs. (A) Representative images of PFKP-staining of human ICC surgical specimens. Scale bars, $500 \mu \mathrm{m}$. (B) PFKP protein expression in human ICC surgical specimens and association with IDH mutation status.

(Fig. 5B). Considering the significance of PFKP for growth advantage in many human cancers ${ }^{29,30}$, PFKP could serve as a novel metabolic target for IDH1-mutant ICCs.

IDH1 mutation provides EGF- and HGF-independent survival in IBOs. We sought to assess the biological consequences of mutant IDH1-induced glycolytic activation. Consistent with previous reports showing that mutant IDH1 alone is insufficient for transformation in several cell linages ${ }^{16,20}$, subcutaneous inoculation of either wt-IBO or mut-IBO into nude mice failed to generate tumors (2,000,000 cells per group, $n=6$, data not shown). The organoid culture systems are applied to the assessment of extracellular factor-dependency ${ }^{31}$. Based on the findings that cultured organoids gradually become independent of growth factors followed by oncogenic mutations $^{31}$, we evaluated the effect of IDH1 mutation for growth factor-dependency in the survival of IBOs. Depletion of EGF, FGF10, HGF, or R-spondin-1 significantly reduced the organoid-forming efficiency of wt-IBO (Supplementary Fig. 5A,B). Although the efficiency of mut-IBO formation decreased in the absence of EGF, FGF10, HGF, or R-spondin-1, the higher organoid-forming efficiency compared to wt-IBO was still observed 
A

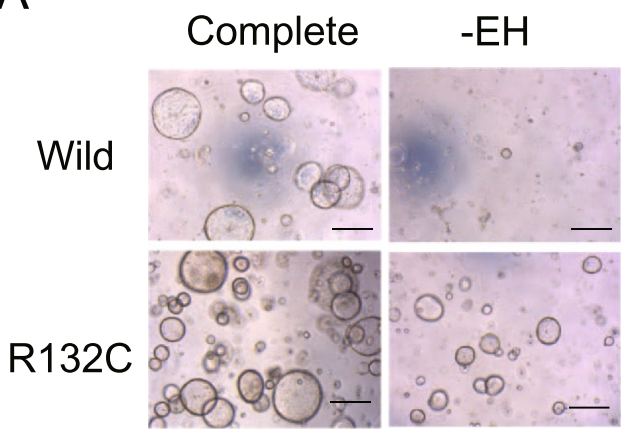

B

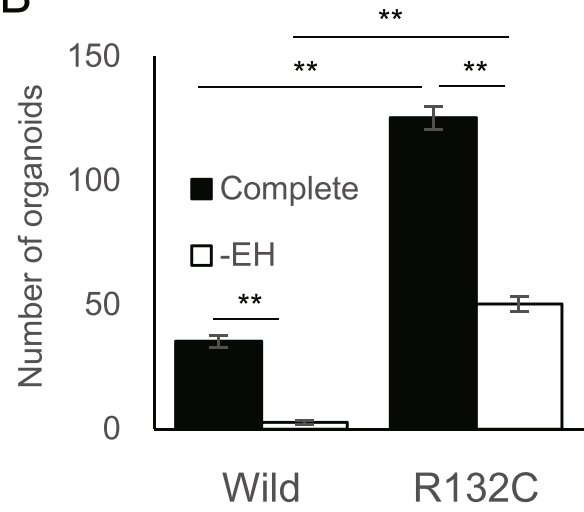

Figure 6. IDH1 mutation overrides the dependency on EGF and HGF in IBOs. (A) Representative images of IBOs at $5 \mathrm{~d}$ after plating (10,000 cells per well), in the presence (Complete) or absence (-EH) of EGF and HGF. Scale bars, $500 \mu \mathrm{m}$. (B) The number of organoids $(>100 \mu \mathrm{m})$ in each group $(\mathrm{n}=3, * * \mathrm{P}<0.01)$.

(Supplementary Fig. 5A,B). Remarkably, double depletion of EGF and HGF (EH-depletion), completely blunted the formation of wt-IBOs, whereas mut-IBOs persisted (Fig. 6A,B).

Mutant IDH1-induced AMPK activation makes IBOs override the EGF and HGF dependency. To address the molecular mechanisms underlying the sustained survival after EH-depletion, we examined the status of signal transduction pathways related to cell survival (Fig. 7A and Supplementary Fig. 6). The levels of phosphorylation of Erk, Akt, or Stat3 were comparable between wt-IBOs and mut-IBOs (Fig. 7A). In addition, these phosphorylation levels were not affected by EH-depletion (Fig. 7A). In contrast, we identified enhanced phosphorylation of AMP-activated protein kinase (AMPK) in mut-IBOs compared to wt-IBOs, regardless of EH-depletion (Fig. 7A). The two primary upstream regulators of AMPK, Lkb1, and Camkk2 were not differently activated between mut-IBOs and wt-IBOs (Supplementary Fig. 7). Intriguingly, among the multiple downstream targets of the AMPK signaling pathway ${ }^{32}$, Unc-51 like kinase-1 (Ulk1), which is a crucial initiator of autophagy ${ }^{33}$, was activated by EH-depletion only in mut-IBOs (Fig. 7A and Supplementary Fig. 7). To address whether AMPK activation contributed to the sustained formation of IBOs under EH-depletion, mut-IBOs were treated with the AMPK inhibitor, Compound $\mathrm{C}^{34}$. The organoid-forming capacity after EH-depletion in mut-IBOs was entirely inhibited by Compound C (Fig. 7B,C), suggesting that AMPK contributes to the growth-factor independence in cooperation with IDH1 mutation. Given that 5-aminoimidazole-4-carboxamide-1- $\beta$-D-ribofuranoside (AICAR), which is a well-known AMP analog that activates AMPK, inhibited the formation of wt-IBOs (Supplementary Fig. 8). Thus, the role of AMPK seems to be context-dependent, as previously reported ${ }^{35}$.

It is well-known that growth factor deprivation, as well as detachment from the extracellular matrix (ECM), induces ATP loss and subsequent cell death in normal epithelial cells ${ }^{36}$. When organoids are passaged as dissociated single cells, they must be exposed to energy stress without growth factors or ECM. Supporting this notion, wt-IBOs were not formed in the settings without matrigel after passage procedures. Thus, we compared the levels of cellular ATP in dissociated IBO cells just after the passage procedure (Fig. 7D,E). Mounting of the dissociated wt-IBO cells on matrigel with complete medium after passage recovered ATP production in a time-dependent manner, but EH-depletion from medium inhibited the recovery (Fig. 7E). In contrast, the dissociated mut-IBO cells restored the high levels of ATP after passage, even under EH-depletion (Fig. 7E). Given the function of AMPK in replenishing ATP after the loss of ECM attachment ${ }^{36}$, we considered that AMPK activation contributed to ATP production in dissociated mut-IBO cells. Consistently, AMPK inhibition significantly decreased ATP levels in the dissociated mut-IBO cells (Fig. 7F). These data supported the notion that AMPK activity contributes to ATP production under EH-depletion in mut-IBOs.

\section{Discussion}

In this study, we found a unique metabolic status in the IDH- mutant biliary cells. Several previous reports have described the phenotype of IDH-mutant cancer cells related to hypoxia-inducible factor 1 (HIF-1) activity. For example, the survival and proliferation of IDH-mutant glioma cells were promoted by the Warburg effect due to the stabilization of HIF- $1 \alpha^{37}$. In contrast, other studies have shown that IDH mutations downregulate the HIF-1 pathway and induce limited glycolysis, contributing to the slower growth and better prognosis in glioma cells ${ }^{38}$. Although we do not entirely exclude the possible involvement of Hif-1 $\alpha$ in the IDH- mutant biliary cells, the expression of Hif- $1 \alpha$ protein and the majority of its downstream glycolytic genes were not markedly upregulated in mut-IBOs (Supplementary Fig. 9). Upregulated HIF-1 $\alpha$ expression by IDH1 mutation reduced the energy flux through the TCA cycle and increased the clonogenicity of normal human astrocytes due to the activation of pyruvate dehydrogenase kinases (PDKs) ${ }^{39}$. In contrast, levels of some metabolites in the TCA cycle, including citrate, fumarate, and malate were elevated in mut-IBOs (Supplementary Fig. 2). Additionally, decreased levels of glutamate identified in glioma or astrocytes harboring $I D H 1$ mutation ${ }^{40}$ were not observed in the case of mut-IBOs (Supplementary Fig. 2). These findings propose a possibility that metabolic rewiring traits triggered by IDH mutation are different among distinct cell lineages. Furthermore, while mutant IDH in patient material 
A

Wild R132C

$(+) \quad(-) \quad(+) \quad(-)$

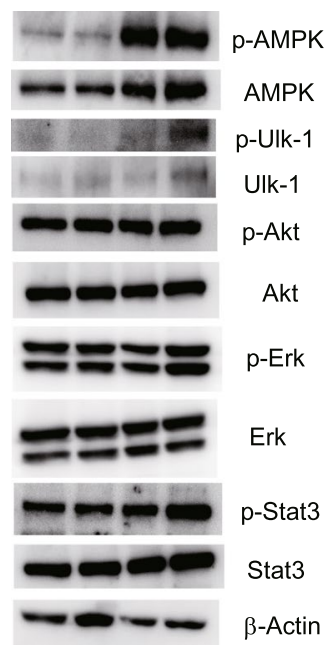

B

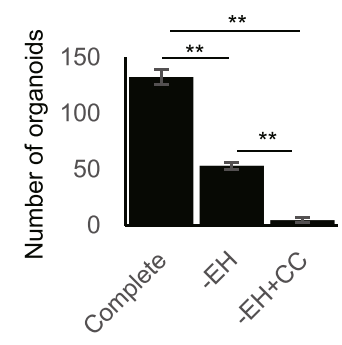

C

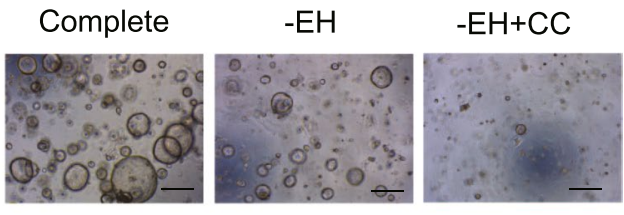

D

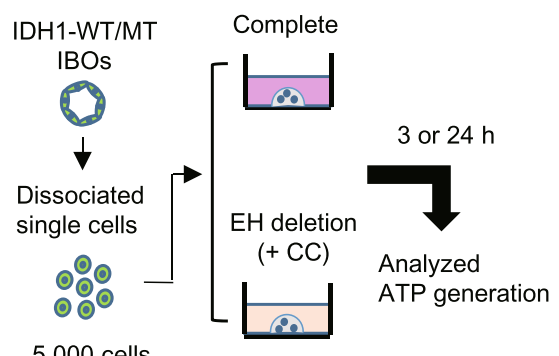

$\mathrm{E}$

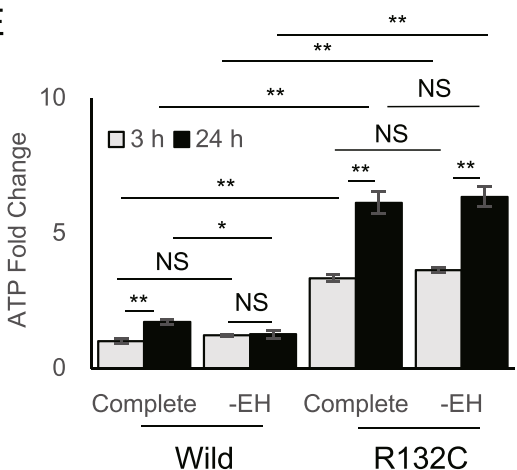

$\mathrm{F}$

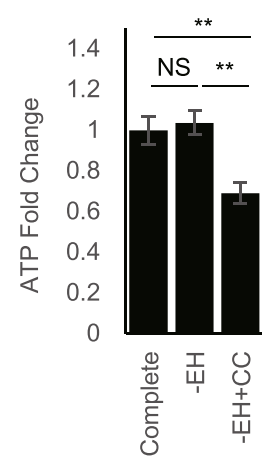

Figure 7. Mutant IDH1-induced growth factor independence is supported by AMPK activation. (A) Immunoblots of AMPK, phosphorylated AMPK (p-AMPK), Ulk1, phosphorylated-Ulk1 (p-Ulk1), Erk, phosphorylated Erk (p-Erk), Akt, phosphorylated Akt (p-Akt), Stat3 and phosphorylated Stat3 (p-Stat3), in IBOs. (B) The number of mut-IBOs $(>100 \mu \mathrm{m})$ at $5 \mathrm{~d}$ after plating $(\mathrm{n}=5,10,000$ cells per group, $* * \mathrm{P}<0.01)$. (C) Representative images. Scale bars, $500 \mu \mathrm{m}$. (D) Schema of experiments analyzing ATP production in dissociated IBO cells. (E) ATP levels of dissociated IBO cells at $24 \mathrm{~h}$ after plating $(5,000$ cells per group, $\mathrm{n}=4$, $* \mathrm{P}<0.05, * * \mathrm{P}<0.01$, NS not significant). (F) ATP levels of dissociated mut-IBO cells at $24 \mathrm{~h}$ after plating $(5,000$ cells per group), in the presence (Complete) or absence (-EH) of EGF and HGF, treated with vehicle or $5 \mu \mathrm{M}$ Compound C (CC) $(\mathrm{n}=5, * * \mathrm{P}<0.01$, NS not significant).

of glioma and glioblastoma was associated with a non-Warburg phenotype in comparison with wild-type IDH tumors, the artificial introduction of mutant IDH into glioblastoma cell lines resulted in aerobic glycolysis ${ }^{41-43}$. Thus, the metabolic effects of mutant IDH can also vary between patient-derived materials and those established experimentally. To assess the possibility of clinical trials focusing on the metabolic traits of mutant IDH tumors as in gliomas ${ }^{44}$, the metabolome analysis of clinical samples of ICC would be needed in the near future.

It was noteworthy that there was a difference in the organoid-forming efficiency between mut-IBOs and wt-IBOs treated with 2-HG (Fig. 1D-F). We speculate two possibilities for the different extent of growth promotion. The first is a possibility that the intracellular concentration of 2-HG in wt-IBO cells treated with 2-HG was lower than in mut-IBOs due to the limited cell permeability of R-2-HG or the run-in effect ${ }^{17}$. The second is a possible 2-HG independent function of mutant IDH1, which might be supported by a previous paper showing that DNA methylation profiles of 2-HG treated cells could not entirely recapitulate those of mutant IDH1 cells ${ }^{45}$.

In general, as the increasing level of AMP mirrors the decrease in ATP under metabolic stress conditions in normal cells ${ }^{32}$, the metabolic situation caused by IDH1 mutation seems to be extraordinary, where both AMP and ATP accumulated. Similarly, the activation of AMPK in mut-IBOs was rather paradoxical in terms of its energy sensor function ${ }^{32}$ because ATP levels remained unperturbed in mut-IBOs (Fig. 2). As the AMP-induced activation of AMPK is allosterically much more potent than the inhibitory action of ATP based on structural research ${ }^{46}$, AMPK activation in mut-IBOs was likely to be induced by AMP accumulation (Fig. 2).

Despite the tumor-suppressive roles of AMPK in many cancers, recent studies have shown the pleiotropic properties of AMPK for tumor cell survival under metabolic stress ${ }^{35}$. In terms of the relationships of AMPK and 
Intrahepatic bile duct
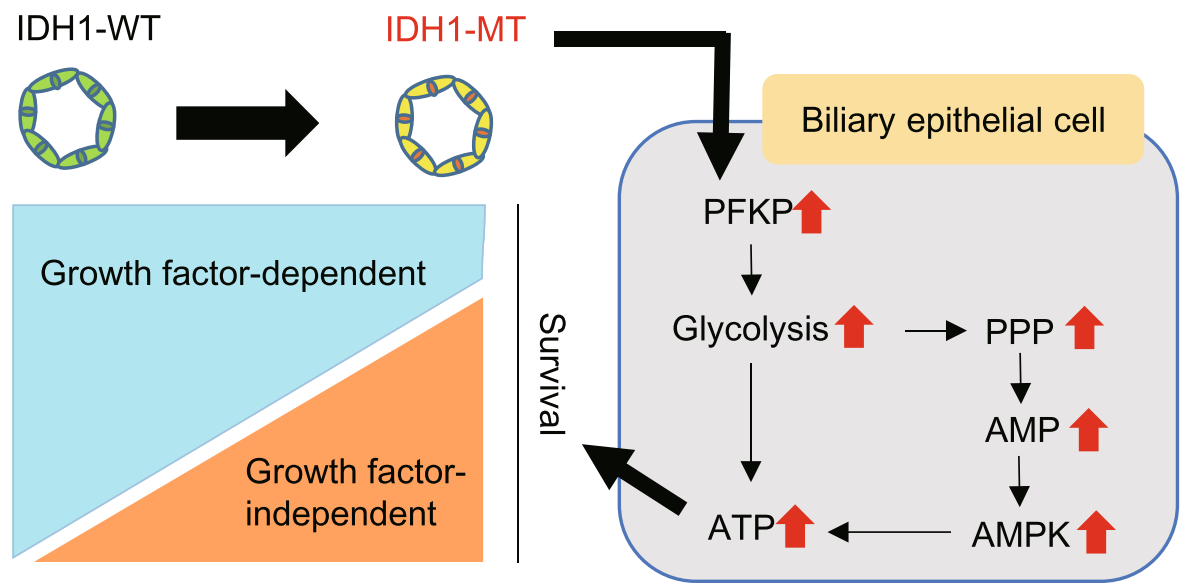

Figure 8. Schematic model of mutant IDH1-induced acquisition of growth factor independence via PFKP upregulation and AMPK activation in intrahepatic biliary epithelial cells.

IDH mutation, previous reports demonstrated the metabolic vulnerability of mutant IDH gliomas or glioblastomas to biguanides, such as metformin and phenformin, which activate AMPK by inhibiting mitochondrial respiratory chain complex $1^{47-49}$. Therefore, our findings emphasize the notion that the role of AMPK seems to be pleiotropic and context-dependent ${ }^{35}$.

Finally, we identified a two-way metabolic rewiring status; PFKP upregulation and AMPK activation induced by IDH1 mutation in normal biliary cells (Fig. 8). Nonetheless, it is still necessary to elucidate the whole mechanism acquired by IDH1 mutation that finally accomplishes biliary tumor development.

\section{Methods}

Liver organoid culture. Organoids derived from murine liver were established according to published protocols ${ }^{24}$. Resected mouse liver was minced under sterile conditions, washed in Hank's balanced salt solution without $\mathrm{Ca}^{+}$and $\mathrm{Mg} 2^{+}$(HBSS-) containing $10 \mathrm{mM}$ HEPES, $0.5 \mathrm{mM}$ EDTA, and then naturally sedimented. After removal of the supernatant, the minced liver was digested in HBSS + containing $10 \mathrm{mM} \mathrm{HEPES,} 1 \mathrm{mg} / \mathrm{mL}$ of collagenase type 4 (Invitrogen, Carlsbad, CA) and $0.5 \mathrm{mg} / \mathrm{mL}$ of pronase (Sigma-Aldrich, St. Louis, MO, USA) for 10-15 min. The suspension was filtered through cell strainers $(100 \mu \mathrm{m})$ and sedimented by centrifugation $(700 \times g)$ for $3 \mathrm{~min}$. After hemolytic treatment, the pellet was mixed with Matrigel (BD Bioscience, San Jose, CA) and cultured as previously described ${ }^{24}$. Culture medium was based on Advanced DMEM/F12 (Invitrogen) supplemented with B27 (Invitrogen), N2 (InvivoGen, San Diego, CA), 1.25 $\mu \mathrm{M}$ N-acetylcysteine (Wako, Osaka, Japan), $50 \mathrm{ng} / \mathrm{mL}$ EGF (Wako), $100 \mathrm{ng} / \mathrm{mL}$ FGF10 (Wako), $50 \mathrm{ng} / \mathrm{mL}$ HGF (R\&D Systems, Minneapolis, MN), $10 \%$ RSPO1-conditioned medium (kindly gifted from C. Kuo and prepared as previously reported ${ }^{23}$ ), $10 \mathrm{nM}$ gastrin (Sigma-Aldrich), and $10 \mathrm{mM}$ nicotinamide (Sigma-Aldrich). For the first $4 \mathrm{~d}$ after seeding, the cells were also supplemented with $100 \mathrm{ng} / \mathrm{mL}$ Noggin (PeproTech, Rocky Hill, NJ) and 10\% Wnt3a-conditioned medium (prepared as previously reported ${ }^{22}$ ). Next, 1 week after seeding, organoids were mechanically dissociated and transferred to fresh gels.

Construction of IDH1 mutation and lentiviral infection. Human wild-type IDH1 expression vector (plasmid pFN21AE0433) was purchased from Kazusa DNA Research Institute (Chiba, Japan). The IDH1 R132C mutant was generated from the wild-type IDH1 open reading frame of this plasmid using the QuikChange Site-Directed Mutagenesis Kit (Agilent Technologies, Santa Rosa, CA) and primers 5'-GATCCCCA TAAGCATGACAACCTATGATGATAGGTTC-3' for sense and 5' - GAACCTATCATCATAGGTTG TCATGCTTATGGGGATC- $3^{\prime}$ for antisense. The wild-type and mutant IDH1 were ligated into the multiple cloning sites of pLVSIN-EF1 $\alpha$ Vector (TAKARA, Shiga, Japan) and these lentiviral vectors were transfected into $293 \mathrm{~T} / 17$ cells. Organoids were infected with concentrated lentivirus supernatant for $9-12 \mathrm{~h}$ and selected with puromycin $(2 \mu \mathrm{g} / \mathrm{mL})$.

Transduction was validated by reverse transcriptase-polymerase chain reaction (RT-PCR) using specific primers for mutant IDH1 (R132C): 5'-GGGTAGAACCTATCATCATAGGTT-3', 5'-TCCAGGCCCAGGAA CAACAA-3' , and wild-type IDH1: 5' - GGGTAGAACCTATCATCATAGGTC-3' $5^{\prime}$ - TCCAGGCCCAGGAAC AACAA- $3^{\prime}$.

shRNA. Lentiviral (pLKO.1) Pfkp shRNA vectors TRCN0000025916 and TRCN0000025962 were purchased from Dharmacon Inc. (Lafayette, CO). and used as shPfkp-2 and shPfkp-5, respectively. The puromycin-resistance gene was excised with restriction enzymes, and the hygromycin-resistance gene was ligated into the same region of each vector. 
Capillary electrophoresis-mass spectrometry (CE-MS). Organoids were collected through complete dilution of gels by Cell Recovery Solution (BD Bioscience, San Jose, CA, USA) and washed twice with 5\% mannitol in order to extract the metabolites. Methanol containing $25 \mu \mathrm{M}$ each of methionine sulfone, 2-(N-morpholino) ethanesulfonic acid (MES), and (+)-Camphor-10-sulfonic acid was added to cell pellets, which were left to rest for $10 \mathrm{~min}$ at room temperature. Samples in solution were mixed with water and chloroform, centrifuged at $10,000 \times g$ for $3 \mathrm{~min}$ at $4^{\circ} \mathrm{C}$, and the aqueous phase of the solution was removed. The extract was transferred to a $5 \mathrm{kDa}$ ultrafiltration tube (Human Metabolome Technologies, Yamagata, Japan) and centrifuged at 9,100 $\times g$ for $2 \mathrm{~h}$ at $20^{\circ} \mathrm{C}$. CE-MS was used to analyze the filtrate in Dr. Soga's laboratory, as previously described ${ }^{50}$.

Transcriptional analysis. GENE CHIP Expression Analysis microarrays were performed following the manufacturer's protocol (Affymetrix, Santa Clara, CA, USA). Total RNA was extracted from murine liver organoids expressing human wild type IDH1 and mutant IDH1 (R132C), at passage three after transfection.

Animals. All experiments were performed under protocols approved by the Animal Ethics Committee of The University of Tokyo. The research was conducted with guidelines approved by the Office of Laboratory Animal Welfare (Ref: F18-00412). C57BL/6 J mice were purchased from CLEA (Shizuoka, Japan).

Products. AGI-5198 was purchased from Cayman Chemical (Ann Arbor, MI). R(-)-2-hydroxyglutarate (2-HG) and 2-deoxy-d-glucose (2DG) were purchased from Sigma-Aldrich. Cell Titer-Glo was purchased from Promega (Madison, WI). Compound C (ab146597) was purchased from Abcam (Cambridge, UK). DMEM/F12 without Glucose (L0091) was purchased from Biowest (Nuaille, France).

Clinical samples, molecular analysis, and PFKP scoring system. Formalin-fixed, paraffin-embedded (FFPE) tissue blocks from 101 patients with ICC who underwent surgical treatment at the University of Tokyo Hospital were used for the extraction of tumor DNA. Mutational analysis of IDH was performed by direct sequencing of PCR products. FFPE tissue sections were immunohistochemically stained using the primary antibody against PFKP (ab119796, Abcam). Staining results were scored by two pathologists (K.M. and A.H.) blinded to the mutational profile of $I D H$. The scoring system was validated by the percentages of positive cells as follows: score $0,<1 \%$; score $1,1-5 \%$; score $2,6-25 \%$; score 3, 26-50\%; and score $4,>51 \%$. Simultaneously, scores 0 and 1 were defined as negative expression, score 2 as low, score 3 as moderate, and score 4 was defined as high expression, respectively. Detailed procedures for immunohistochemistry and DNA sequencing were previously described ${ }^{51}$. This study was approved by the Ethics Committee of the University of Tokyo, and carried out in accordance with the Declaration of Helsinki. Informed consent in writing was obtained from all patients.

PFK-1 activity assay. 6-Phosphofructokinase Activity Assay Kit (ab155898, Abcam) was used in accordance with the manufacturer's protocol. Briefly, for each assay, 900,000 cells were harvested and washed in cold PBS. After suspension and appropriate dilution in the assay buffer, cellular extracts were mixed with the reaction solution containing ATP and F6P. NADH produced by the reaction was monitored spectrophotometrically at $450 \mathrm{~nm}$ for $100 \mathrm{~min}$. PFK-1 activity was calculated from an NADH standard curve.

Glucose uptake assay. Glucose Uptake Assay Kit (ab136955, Abcam) was used in accordance with the manufacturer's protocol. Briefly, organoids were treated in Krebs-Ringer-Phosphate-Hepes (KRPH) buffer: $20 \mathrm{mM}$ HEPES, $5 \mathrm{mM} \mathrm{KH} 2 \mathrm{PO} 4,1 \mathrm{mM} \mathrm{MgSO} 4,1 \mathrm{mM} \mathrm{CaCl} 2,136 \mathrm{mM} \mathrm{NaCl}$, and $4.7 \mathrm{mM} \mathrm{KCl}$, at a pH $7.4 \mathrm{con}-$ taining $2 \%$ bovine serum albumin (BSA), with or without 2-DG $(1 \mathrm{mM})$, for $20 \mathrm{~min}$. Organoids were harvested and washed in cold PBS. For each assay, we used 70,000 cells. Harvested cells were lysed with extraction buffer, frozen at $-80^{\circ} \mathrm{C}$ for $15 \mathrm{~min}$, and heated at $85^{\circ} \mathrm{C}$ for $40 \mathrm{~min}$. After cooling on ice for $5 \mathrm{~min}$, the lysates were neutralized by adding neutralization buffer and diluted appropriately with assay buffer. Lastly, by two amplification steps, according to the manufacturer's instructions, the colorimetric end product was detected at $412 \mathrm{~nm}$. From the 2-DG-6-phosphate (2-DG6P) standard curve, 2-DG (or glucose) uptake was calculated.

Quantitative real-time reverse transcriptase-PCR (RT-qPCR). RT-qPCR was performed as previously described ${ }^{52}$. RNA was extracted from organoids using RNeasy Mini Kit (Qiagen, Hilden, Germany). Values were internally normalized against $\beta$-actin $(A c t b)$ mRNA expression. PCR primer sequences are listed in Supplementary Table 2 .

Immunohistochemistry. Organoids were fixed in 4\% paraformaldehyde (PFA) in PBS. The slides were hematoxylin \& eosin (H\&E) stained and subjected to histological analysis. Immunohistochemistry was performed as previously described ${ }^{52}$. The primary antibodies used were anti-Ck19 (DSHB, AB-2133570, 1:100) and anti-Sox9 (Sigma-Aldrich, AB5535, 1:1000).

Immunoblotting. Harvested organoids were lysed in RIPA buffer [10 mM Tris-HCl (pH 7.4)], $150 \mathrm{mM}$ $\mathrm{NaCl}, 2 \mathrm{mM}$ EDTA, $1 \% \mathrm{NP} 40,0.1 \% \mathrm{Na}$ deoxycholate, $0.1 \%$ SDS, $50 \mathrm{mM} \mathrm{NaF}, 1 \mathrm{mM} \mathrm{Na} 3 \mathrm{VO}$, and a protease inhibitor cocktail (Complete Mini, Roche, Basel, Switzerland), then sonicated for $5 \mathrm{~min}$. After centrifugation at $15,000 \times g$ for $15 \mathrm{~min}$, supernatants were collected as whole cell lysates. Immunoblotting was performed as previously described ${ }^{52}$. The primary antibodies used were anti- $\beta$-actin (Sigma-Aldrich, A5441, 1:10,000), anti-Akt (Cell Signaling, 9272, 1:1,000), anti-phosphorylated-Akt (Cell Signaling, 9271, 1:1,000), anti-Histone H3 (Abcam, ab1791, 1:1,000), anti-H3K9me3 (Abcam, ab8898, 1:1,000), anti-H3K9me1 (Abcam, ab9045, 1:1,000), anti-H3K4me3 (Abcam, ab8580, 1:1,000), anti-H3K27me3 (Abcam, ab6002, 1:1,000), anti-Pfkp (Abcam, ab119796, 1:500), anti-Pfkl (Abcam, ab37583, 1:500), anti-Pfkm (Proteintech, 55028-1-AP, 1:800), anti-Erk (Cell Signaling, 9102, 1:1000), anti- phosphorylated Erk (Cell Signaling, 9101, 1:1000), anti-Ampk (Cell 
Signaling, 2532, 1:1000), anti- phosphorylated Ampk (Cell Signaling, 2535, 1:1000), anti-Hif-1 $\alpha$ (Abcam, ab463, 1:1000), anti-Lkb1 (Cell Signaling, 3047, 1:1000), anti-phosphorylated-Lkb1 (Cell Signaling, 3482, 1:1000), anti-Camkk2 (Cell Signaling, 16810, 1:1000), anti-phosphorylated-Camkk2 (Cell Signaling, 12818, 1:1000), anti-Pfkfb3 (Abcam, ab181861, 1:1000), anti-phosphorylated-Pfkfb3 (Abcam, ab202291, 1:1000), anti-p70S6k (Cell Signaling, 2708, 1:1000), anti-phosphorylated-p70S6k (Cell Signaling, 9205, 1:1000), anti-Ulk1 (Cell Signaling, 8054, 1:1000), anti-phosphorylated-Ulk1 (Cell Signaling, 5869, 1:1000), anti-Beclin1 (Cell Signaling, 3495, 1:1000), anti-phosphorylated-Beclin1 (Cell Signaling, 14717, 1:1000), anti-Raptor (Cell Signaling, 2280, 1:1000), anti- phosphorylated- Raptor (Cell Signaling, 2083, 1:1000), anti-ACC (Cell Signaling, 3676, 1:1000), and anti- phosphorylated- ACC (Cell Signaling, 11818, 1:1000).

Dot blot analysis. Genomic DNA samples were collected from organoids using QIAamp DNA Mini Kit (Qiagen). Dot blot analysis was performed as described previously ${ }^{53}$. The primary antibodies used were anti-5-hydroxymethylcytosine (5-hmC) $(39769,1: 10,000$, Active Motif, Carlsbad, CA, USA) and anti-5-Methylcytosine (5-mC) (NA81, 1:1,000, Merck Millipore, Burlington, MA, USA).

ATAC-seq. ATAC-seq experiments were performed on 100,000 cells from the organoid samples, following published protocols ${ }^{27}$. We prepared the samples from wt-/mut-IBOs, at passage 8. Processing of ATAC-Seq reads were performed as follows. Sequence reads were trimmed and aligned to the mouse genome (mm10) using $\mathrm{BWA}^{54}$. PCR duplicates were removed using Picard's MarkDuplicates, and peaks were called using MACS2 ${ }^{55}$. Called peaks were further processed to obtain scatter-plots, correlation heat maps, and Venn diagrams using DiffBind ${ }^{27}$.

Chromatin immunoprecipitation (ChIP). ChIP was performed as previously described ${ }^{52,56}$. We prepared $1.5 \times 10^{6}$ cells from liver organoids expressing human wild type IDH1 and mutant IDH1 for each assay. Antibodies used for ChIP were H3K4me3 (ab8580, Abcam) and normal Rabbit IgG (CST2729, Cell Signaling Technology, Danvers, MA). ChIP primers are listed in Supplementary Table 3.

Statistical analysis. Data are presented as means \pm standard error of the mean (SEM). Statistical significance was evaluated by the two-tailed Student's $t$-test. * denotes $\mathrm{P}<0.05$, and $* * \mathrm{P}<0.01$. To test the correlation between PFKP expression and IDH status in human ICC samples, Fisher's exact test was used.

\section{Data availability}

The datasets analyzed in the current study are available from the corresponding author on reasonable request. Microarray gene expression data are in Gene Expression Omnibus (GSE134760).

Received: 14 September 2019; Accepted: 22 November 2019;

Published online: 11 December 2019

\section{References}

1. Rizvi, S. \& Gores, G. J. Pathogenesis, Diagnosis, and Management of Cholangiocarcinoma. Gastroenterology 145, 1215-1229 (2013).

2. Everhart, J. E. \& Ruhl, C. E. Burden of digestive diseases in the United States Part III: Liver, biliary tract, and pancreas. Gastroenterology 136, 1134-44 (2009).

3. Tyson, G. L. \& El-Serag, H. B. Risk factors for cholangiocarcinoma. Hepatology 54, 173-184 (2011).

4. Zou, S. et al. Mutational landscape of intrahepatic cholangiocarcinoma. Nature Communications 5, 5696 (2014).

5. Fujimoto, A. et al. Whole-genome mutational landscape and characterization of noncoding and structural mutations in liver cancer. Nat Genet 48, 500-509 (2016).

6. Borger, D. R. et al. Frequent Mutation of Isocitrate Dehydrogenase (IDH) 1 and IDH2 in Cholangiocarcinoma Identified Through Broad-Based Tumor Genotyping. The Oncologist 17, 72-79 (2012).

7. Chan-on, W. et al. Exome sequencing identifies distinct mutational patterns in liver fluke-related and non-infection-related bile duct cancers. Nat Genet 45, 1474-1478 (2013).

8. Lowery, M. A. et al. Comprehensive Molecular Profiling of Intrahepatic and Extrahepatic Cholangiocarcinomas: Potential Targets for Intervention. Clin Cancer Res 24, 4154-4161 (2018).

9. Nepal, C. et al. Genomic perturbations reveal distinct regulatory networks in intrahepatic cholangiocarcinoma. Hepatology $\mathbf{6 8}$, 949-963 (2018).

10. Farshidfar, F. et al. Integrative Genomic Analysis of Cholangiocarcinoma Identifies Distinct IDH-Mutant Molecular Profiles. Cell Rep 19, 2878-2880 (2017).

11. Cairns, R. A. \& Mak, T. W. Oncogenic Isocitrate Dehydrogenase Mutations: Mechanisms, Models, and Clinical Opportunities. Cancer Discovery 3, 730-741 (2013).

12. Dang, L. et al. Cancer-associated IDH1 mutations produce 2-hydroxyglutarate. Nature 462, 739-744 (2009).

13. Chowdhury, R. et al. The oncometabolite 2-hydroxyglutarate inhibits histone lysine demethylases. EMBO reports 12, $463-469$ (2011).

14. Xu, W. et al. Oncometabolite 2-Hydroxyglutarate Is a Competitive Inhibitor of $\alpha$-Ketoglutarate-Dependent Dioxygenases. Cancer cell 19, 17-30 (2011).

15. Lu, C. et al. IDH mutation impairs histone demethylation and results in a block to cell differentiation. Nature 483, 474-478 (2012).

16. Sasaki, M. et al. IDH1(R132H) mutation increases murine haematopoietic progenitors and alters epigenetics. Nature 488, 656-659 (2012).

17. Turcan, S. et al. IDH1 mutation is sufficient to establish the glioma hypermethylator phenotype. Nature 483, 479-483 (2012).

18. Fan, B. et al. Cholangiocarcinomas can originate from hepatocytes in mice. The Journal of Clinical Investigation 122, 2911-2915 (2012).

19. Guest, R. V. et al. Cell Lineage Tracing Reveals a Biliary Origin of Intrahepatic Cholangiocarcinoma. Cancer Research 74, 1005-1010 (2014).

20. Saha, S. K. et al. Mutant IDH inhibits HNF-4[agr] to block hepatocyte differentiation and promote biliary cancer. Nature 513, 110-114 (2014)

21. Sato, T. et al. Single Lgr5 stem cells build crypt-villus structures in vitro without a mesenchymal niche. Nature 459, 262-265 (2009). 
22. Barker, N. et al. Lgr5(+ve) stem cells drive self-renewal in the stomach and build long-lived gastric units in vitro. Cell Stem Cell 6, 25-36 (2010).

23. Farin, H. F., Van Es, J. H. \& Clevers, H. Redundant sources of Wnt regulate intestinal stem cells and promote formation of Paneth cells. Gastroenterology 143, 1518-1529.e7 (2012).

24. Huch, M. et al. In vitro expansion of single Lgr5+ liver stem cells induced by Wnt-driven regeneration. Nature 494, 247-250 (2013).

25. Rohle, D. et al. An inhibitor of mutant IDH1 delays growth and promotes differentiation of glioma cells. Science 340, 626-30 (2013).

26. Warburg, O. On the Origin of Cancer Cells. Science 123, 309-314 (1956).

27. Buenrostro, J. D., Giresi, P. G., Zaba, L. C., Chang, H. Y. \& Greenleaf, W. J. Transposition of native chromatin for fast and sensitive epigenomic profiling of open chromatin, DNA-binding proteins and nucleosome position. Nat Methods 10, 1213-8 (2013).

28. Dunaway, G. A., Kasten, T. P., Sebo, T. \& Trapp, R. Analysis of the phosphofructokinase subunits and isoenzymes in human tissues. Biochem J 251, 677-83 (1988).

29. Wang, J. et al. The platelet isoform of phosphofructokinase contributes to metabolic reprogramming and maintains cell proliferation in clear cell renal cell carcinoma. Oncotarget 7, 27142-57 (2016).

30. Lee, J. H. et al. Stabilization of phosphofructokinase 1 platelet isoform by AKT promotes tumorigenesis. Nat Commun 8, 949 (2017).

31. Fujii, M. et al. A Colorectal Tumor Organoid Library Demonstrates Progressive Loss of Niche Factor Requirements during Tumorigenesis. Cell Stem Cell 18, 827-38 (2016).

32. Garcia, D. \& Shaw, R. J. AMPK: Mechanisms of Cellular Energy Sensing and Restoration of Metabolic Balance. Mol Cell 66, 789-800 (2017).

33. Wong, P. M., Puente, C., Ganley, I. G. \& Jiang, X. The ULK1 complex: sensing nutrient signals for autophagy activation. Autophagy 9, 124-37 (2013).

34. Zhou, G. et al. Role of AMP-activated protein kinase in mechanism of metformin action. The Journal of clinical investigation 108, $1167-1174(2001)$.

35. Zadra, G., Batista, J. L. \& Loda, M. Dissecting the Dual Role of AMPK in Cancer: From Experimental to Human Studies. Mol Cancer Res 13, 1059-72 (2015).

36. Buchheit, C. L., Weigel, K. J. \& Schafer, Z. T. Cancer cell survival during detachment from the ECM: multiple barriers to tumour progression. Nat Rev Cancer 14, 632-41 (2014).

37. Zhao, S. et al. Glioma-derived mutations in IDH1 dominantly inhibit IDH1 catalytic activity and induce HIF-1alpha. Science 324, 261-5 (2009).

38. Chesnelong, C. et al. Lactate dehydrogenase A silencing in IDH mutant gliomas. Neuro-Oncology 16, 686-695 (2014).

39. Izquierdo-Garcia, J. L. et al. IDH1 Mutation Induces Reprogramming of Pyruvate Metabolism. Cancer Res 75, 2999-3009 (2015).

40. Reitman, Z. J. et al. Profiling the effects of isocitrate dehydrogenase 1 and 2 mutations on the cellular metabolome. Proceedings of the National Academy of Sciences 108, 3270-3275 (2011).

41. Nie, Q. et al. Overexpression of isocitrate dehydrogenase-1R(1)(3)(2)H enhances the proliferation of A172 glioma cells via aerobic glycolysis. Mol Med Rep 11, 3715-21 (2015).

42. Lenting, K. et al. Isocitrate dehydrogenase 1-mutated human gliomas depend on lactate and glutamate to alleviate metabolic stress. Faseb j 33, 557-571 (2019).

43. Khurshed, M., Molenaar, R. J., Lenting, K., Leenders, W. P. \& van Noorden, C. J. F. In silico gene expression analysis reveals glycolysis and acetate anaplerosis in IDH1 wild-type glioma and lactate and glutamate anaplerosis in IDH1-mutated glioma. Oncotarget 8, 49165-49177 (2017).

44. Molenaar, R. J. et al. Study protocol of a phase IB/II clinical trial of metformin and chloroquine in patients with IDH1-mutated or IDH2-mutated solid tumours. BMJ Open 7, e014961 (2017).

45. Chaturvedi, A. et al. Enantiomer-specific and paracrine leukemogenicity of mutant IDH metabolite 2-hydroxyglutarate. Leukemia (2016).

46. Gowans, G. J., Hawley, S. A., Ross, F. A. \& Hardie, D. G. AMP is a true physiological regulator of AMP-activated protein kinase by both allosteric activation and enhancing net phosphorylation. Cell Metab 18, 556-66 (2013).

47. Cuyas, E. et al. Oncometabolic mutation IDH1 R132H confers a metformin-hypersensitive phenotype. Oncotarget 6, 12279-96 (2015).

48. Grassian, A. R. et al. IDH1 Mutations Alter Citric Acid Cycle Metabolism and Increase Dependence on Oxidative Mitochondrial Metabolism. Cancer Research 74, 3317-3331 (2014).

49. Molenaar, R. J. et al. Radioprotection of IDH1-Mutated Cancer Cells by the IDH1-Mutant Inhibitor AGI-5198. Cancer Res 75, 4790-802 (2015).

50. Satoh, K. et al. Global metabolic reprogramming of colorectal cancer occurs at adenoma stage and is induced by MYC. Proc Natl Acad Sci USA 114, E7697-e7706 (2017).

51. Hayashi, A. et al. Distinct Clinicopathologic and Genetic Features of 2 Histologic Subtypes of Intrahepatic Cholangiocarcinoma. The American Journal of Surgical Pathology 40, 1021-1030 (2016).

52. Nakatsuka, T. et al. Impact of histone demethylase KDM3A-dependent AP-1 transactivity on hepatotumorigenesis induced by PI3K activation. Oncogene 36, 6262-6271 (2017).

53. Kudo, Y. et al. Loss of 5-hydroxymethylcytosine is accompanied with malignant cellular transformation. Cancer Sci 103, 670-6 (2012).

54. Li, H. \& Durbin, R. Fast and accurate short read alignment with Burrows-Wheeler transform. Bioinformatics 25, 1754-60 (2009).

55. Zhang, Y. et al. Model-based analysis of ChIP-Seq (MACS). Genome Biol 9, R137 (2008).

56. Yamamoto, K. et al. Stromal remodeling by the BET bromodomain inhibitor JQ1 suppresses the progression of human pancreatic cancer. Oncotarget 7, 61469-61484 (2016).

\section{Acknowledgements}

We thank Mitsuko Tsubouchi and Sayaka Ito for providing assistance with various cell cultures; and all of the lab members for their helpful comments. This project was supported by Grants-in-Aid for Scientific Research (18K07899) and for Early-Career Scientists (19K17391) from the Japan Society for the Promotion of Science.

\section{Author contributions}

H.F. and K.T. conceived study designs. H.F. performed most of the experiments and wrote the manuscript. K.M., A.H., and M.F. validated the profiles of $I D H$ status and P.F.K.P. expression of human I.C.C. samples provided by K.H. K.I. and T.S. performed metabolic analysis. H.K. analyzed ATAC-seq data. T.N., N.S., K.Y., Y.K., Y.H., H.N., Y.T., H.I. (Hideaki Ijichi), H.K., Y.N. and H.I. (Hiroyuki Isayama) provided technical and material supports. K.K. supervised the study. All authors reviewed the manuscript.

\section{Competing interests}

The authors declare no competing interests. 


\section{Additional information}

Supplementary information is available for this paper at https://doi.org/10.1038/s41598-019-55211-w.

Correspondence and requests for materials should be addressed to K.T.

Reprints and permissions information is available at www.nature.com/reprints.

Publisher's note Springer Nature remains neutral with regard to jurisdictional claims in published maps and institutional affiliations.

(c) (i) Open Access This article is licensed under a Creative Commons Attribution 4.0 International License, which permits use, sharing, adaptation, distribution and reproduction in any medium or format, as long as you give appropriate credit to the original author(s) and the source, provide a link to the Creative Commons license, and indicate if changes were made. The images or other third party material in this article are included in the article's Creative Commons license, unless indicated otherwise in a credit line to the material. If material is not included in the article's Creative Commons license and your intended use is not permitted by statutory regulation or exceeds the permitted use, you will need to obtain permission directly from the copyright holder. To view a copy of this license, visit http://creativecommons.org/licenses/by/4.0/.

(c) The Author(s) 2019 